\title{
Transportan-derived cell-penetrating peptide delivers siRNA to inhibit replication of influenza virus in vivo
}

This article was published in the following Dove Medical Press journal:

Drug Design, Development and Therapy

\author{
Cuiling Zhang' \\ Weigang Ren' \\ Qingxin $\mathrm{Liu}^{2}$ \\ Zhikai $\operatorname{Tan}^{3}$ \\ Junwei Li' \\ Chunyi Tong ${ }^{3}$ \\ 'College of Veterinary Medicine, \\ Qingdao Agricultural University, \\ Qingdao 266I09, People's Republic \\ of China; ${ }^{2}$ Jiangsu Vocational College \\ of Agriculture and Forestry, Jurong \\ 212400, People's Republic of China; \\ ${ }^{3}$ Hunan Province Key Laboratory \\ of Plant Functional Genomics and \\ Developmental Regulation, State Key \\ Laboratory of Chemo/Biosensing and \\ Chemometrics, College of Biology, \\ Hunan University, Changsha 410082, \\ People's Republic of China
}

Introduction: In this study, we report on the development of an effective delivery system for siRNAs; a novel cell-penetrating peptide (CPP), T9(dR), obtained from transportan (TP), was used for in vivo and in vitro testing.

Methods: In this study, toxicity of T9(dR) and TP and efficient delivery of siRNA were tested in 293T, MDCK, RAW, and A549 cells. Furthermore, T9(dR)- and TP-delivered siRNAs against nucleoprotein (NP) gene segment of influenza virus ( Results: Gel retardation showed that $\mathrm{T} 9(\mathrm{dR})$ effectively condensed siRNA into nanoparticles sized between 350 and $550 \mathrm{~nm}$ when the mole ratio of T9(dR) to siRNA was $\geq 4: 1$. In vitro studies demonstrated that $\mathrm{T} 9(\mathrm{dR})$ successfully delivered siRNA with low cellular toxicity into several cell lines. It was also observed that $\mathrm{T} 9(\mathrm{dR})$-delivered siRNAs inhibited replication of influenza virus more efficiently as compared to that delivered by TP into the MDCK and A549 cells. It was also noticed that when given a combined tail vein injection of siNP and T9(dR) or $\mathrm{TP}$, all mice in the $50 \mathrm{nmol}$ siNP group infected with PR8 influenza virus survived and showed weight recovery at 2 weeks post-infection.

Conclusion: This study indicates that $\mathrm{T} 9(\mathrm{dR})$ is a promising siRNA delivery tool with potential application for nucleotide drug delivery.

Keywords: cell-penetrating peptide, CPP, siRNA, inhibition, influenza virus, IV, transportan, nucleoprotein, NP

\section{Introduction}

Influenza A virus infection is a leading cause of almost 200,000 hospitalization and 25,000 annual deaths in the Unites States. ${ }^{1,2}$ In addition, influenza A virus is capable of causing pandemics, making the discovery of novel therapeutics against influenza A imperative. ${ }^{3}$ Although vaccination is an effective strategy to prevent influenza virus infection, less coverage of potent vaccination and vaccinal mismatch of seasonal influenza viruses demand serious consideration. Anti-influenza drugs are efficient in treating influenza virus infection, yet they fail to provide complete protection due to frequent emergence of drug-resistant influenza viruses. ${ }^{4}$ Furthermore, anti-influenza drug must be taken within 48 hours after the onset of symptoms to obtain a clinically successful rapid response. ${ }^{5,6}$ Nucleoprotein (NP) of influenza virus is a structural protein which encapsidates the negative-strand viral RNA. Among eight gene segments of type A influenza virus, NP gene is highly conserved. ${ }^{7}$ Therefore, NP may be targeted for the development of genetic therapy for influenza virus infection.

RNAi is a sequence-specific biological process in which short (21-26 nucleotides), double-stranded RNA molecules inhibit the expression of target genes through a series 
of steps after the formation of RNAi-induced silencing complex..$^{8-11}$ In the past decades, RNAi technique was also introduced into antivirus research. An array of siRNAs have been designed to interfere with the expression of gene segments of influenza viruses and have been shown to inhibit the replication of influenza virus in mammalian cells and mouse model. ${ }^{12-14}$ However, with negative charge, it is hard for siRNA to access intracellular active site. To address this issue, a plethora of delivery platforms have been developed. Current delivery tools for siRNA are cationic lipid and virus-based vectors, but the latter has been facing problems when used in clinical trials. Cell-penetrating peptides (CPPs), consisting of a class of highly effective short peptides, are able to penetrate cell membranes and transport different components into cells with high efficiency. ${ }^{15,16}$ When CPPs non-covalently bind to oligonucleotides and form non-covalent nanoparticles, they retain the translocation properties. ${ }^{17-19}$ These short, synthetic peptide-based vectors were considered as biocompatible and economical candidates for the delivery of therapeutic oligonucleotides. ${ }^{20}$ Binding potent agents to CPPs has been suggested to improve their entry into cells and bioavailability. ${ }^{21-23}$ Among the published CPPs, transportan (TP), a 27 amino acid chimeric peptide which is a combination of $\mathrm{N}$ terminal fragment of neuropeptide galanin and membrane interacting wasp venom peptide mastoparan, is an effective cargo delivery tool with low cytotoxicity. ${ }^{24,25}$ Several results have shown that analogs and derivatives of TP can transport small proteins and DNA and RNA oligomers into cells via direct membrane interactions (transient pore formation), ${ }^{26,27}$ however, inefficiently. Arginine-rich cell-penetrating peptide is another kind of delivery implemented for small molecules. ${ }^{17,28-31}$ Based on the above findings, we designed and synthesized a CPP, T9(dR), with a length of 36 amino acids consisting of TP and nona-D-arginine residues $(9(d R))$. In vitro studies showed that $T 9(d R)$ induced efficient transportation of siRNA into a variety of cell lines with low toxicity and high efficiency. This occurred when the cell lines were treated with a complex of T9(dR) and siRNA. In addition, in vitro and in vivo experiments demonstrated that T9(dR) delivered siNP into epithelial cells in respiratory tract and inhibited replication of influenza virus more efficiently than TP. These results showed that T9(dR) is an interesting vector for siRNA delivery which shows an intriguing perspective for therapy of influenza virus infection.

\section{Materials and methods}

\section{Cell culture, influenza virus, and mice}

Madin-Darby Canine Kidney (MDCK) epithelial cells (CCL34; American Type Culture Collection [ATCC], Manassas, VA, USA) were cultured in modified Eagle's medium
(HyClone, Logan, UT, USA) with 10\% fetal calf serum, $1 \mathrm{mM}$ sodium pyruvate, $1 \times$ nonessential amino acids, $5 \mu \mathrm{g} /$ $\mathrm{mL}$ gentamycin sulfate, and $4 \mathrm{mM}$ L-glutamine (Thermo Fisher Scientific, Waltham, MA, USA). A549 cells (CCL185; ATCC), HEK 293T cells (CRL-11268; ATCC), and RAW cells (CRL-2278; ATCC) were cultured in DMEM supplemented with $10 \% \mathrm{FBS}$ and penicillin $(100 \mathrm{U} / \mathrm{mL}) /$ streptomycin $(100 \mu \mathrm{g} / \mathrm{mL})$. PR8 influenza virus (H1N1) was produced and rescued using the influenza viral plasmid system consisting of $\mathrm{pHW}-\mathrm{PB} 2$, $\mathrm{pHW}-\mathrm{PB} 1, \mathrm{pHW}-\mathrm{PA}, \mathrm{pHW}-$ HA, pHW-NP, pHW-NA, pHW-M, and pHW-NS (gifted by Dr Webster), propagated in 10-day-old embryonated eggs (Baisaisi Laboratories, Jinan, People's Republic of China), titrated with MDCK cells, aliquoted, and stored at $-80^{\circ} \mathrm{C}$ for use. $\mathrm{LD}_{50}$ of influenza viruses in mice was determined using the Reed-Muench method. BALB/c mice were purchased from experimental animal center of Shandong Province and housed under specific pathogen-free conditions in the LARC facilities at Qingdao Agricultural University.

\section{Peptide and siRNA}

Published reports stated that TP and $9(\mathrm{dR})$ could deliver functional siRNA into different cell lines. ${ }^{23,25}$ In our study, we designed a chimeric peptide T9(dR) by linking TP and 9(dR). The sequence was GWTLNSAGYLLGKINLKALAALA KKILdRdRdRdRdRdRdRdRdR and synthesized by GenScript (Nanjing, People's Republic of China). Unconjugated siRNA against GFP gene (siGFP) and cy3 (an indicator for uptake of siRNA) conjugated siGFP: sense: 5'-AAGCUGACCCUGAAGUUCAdTdT-3', anti-sense: 5'-UGAACUUCAGGGUCAGCUUdTdT- $3^{\prime} \cdot{ }^{32,33}$ siNP sense: 5'-GGAUCUUAUUUCUUCGGAGdTdT-3' and guide strand: 5'-CUCCGAAGAAAUAAGAUCCdTdT-3' were purchased from GenScript.

\section{Gel retardation assay and nanoparticle size measurement}

A mixture of siRNA and T9(dR) or TP in PBS (pH 7.4) was kept at room temperature for 15 minutes. T9(dR) siRNA binding capability was evaluated by electrophoresis through $2 \%$ agarose in TAE buffer (40 mM Tris$\mathrm{HCl}, 1 \mathrm{v} / \mathrm{v} \%$ acetic acid, and $1 \mathrm{mM}$ EDTA) at $100 \mathrm{~V}$ for 20 minutes. The siRNA bands were stained with ethidium bromide and imaged using a VersaDoc Imaging System (Bio-Rad Laboratories, Inc., Hercules, CA, USA). To test the stability of a complex consisting of CPP and siRNA, $100 \mathrm{nmol}$ siRNA and $400 \mathrm{nmol} \mathrm{T} 9(\mathrm{dR})$ or $1.6 \mu \mathrm{mol} \mathrm{TP}$ were dissolved in $50 \mu \mathrm{L}$ PBS ( $\mathrm{pH} 7.4$ ), respectively, mixed gently, and kept in room temperature for 15,30 , 
and 60 minutes. The size of the complexes was determined at $25^{\circ} \mathrm{C}$ and $37^{\circ} \mathrm{C}$ using the Zetasizer Nano Zeta potential analyzer (Malvern Instruments, Malvern, UK) at 15, 30, and 60 minutes after mixing CPP and siRNA together.

\section{Assessment of CPP cytotoxicity}

The MTT assay, a colorimetric method, with $\operatorname{NAD}(P)$ $\mathrm{H}$-dependent cellular oxidoreductase enzymes being involved, for measuring the activity of mitochondrial succinate dehydrogenase in living cells, was used. In brief, HEK 293T cells were seeded in 96-well plates at a density of $2 \times 10^{4}$ cells/well in $0.2 \mathrm{~mL}$ of medium. After 16 hours, CPPs were kept at $25^{\circ} \mathrm{C}$ for 15 minutes. Then CPPs with different concentrations were added into wells. After 24 hours of incubation, $20 \mu \mathrm{L}$ MTT solution was added into each well to reach the final concentration of $10 \mathrm{mg} / \mathrm{mL}$. After additional incubation for 2 hours, the medium was removed followed by the addition of $100 \mu \mathrm{L}$ dimethylsulfoxide, and absorbance was read in a microplate reader (BioTek, Winooski, VT, USA) at $590 \mathrm{~nm}$ wavelength.

\section{Fluorescence and flow cytometry}

About $2 \times 10^{4}$ A549 cells or $3 \times 10^{4}$ HEK 293 T cells were seeded in an eight-well chamber and incubated at $37^{\circ} \mathrm{C}$ with $5 \% \mathrm{CO}_{2}$ overnight. Subsequently, the cells were treated with a mixture of $4 \mathrm{nmol} \mathrm{T} 9(\mathrm{dR})$ and $1 \mathrm{nmol}$ cy 3 conjugated siGFP or $16 \mathrm{nmol}$ TP and $1 \mathrm{nmol}$ cy3-conjugated siGFP (incubated at room temperature for 15 minutes). At 24 hours post-transfection, the cells in the chamber were fixed in 4\% paraformaldehyde for 30 minutes, washed thrice with $\mathrm{Na}_{3} \mathrm{~N}_{3} / \mathrm{PBS}$, incubated with $50 \mu \mathrm{L}$ saponin for 30 minutes at room temperature, washed thrice with $\mathrm{PBS} / \mathrm{BSA} / \mathrm{Na}_{3} \mathrm{~N}_{3}$, and mounted with DAPI (Southern Bioteck, Birmingham, AL, USA) and observed on microscope (Nikon eclipse, Chiyoda, Japan). About $5 \times 10^{5}$ A549 cells, $8 \times 10^{5}$ HEK $293 \mathrm{~T}$ cells, $8 \times 10^{5}$ RAW cells, and $5 \times 10^{5}$ MDCK cells were seeded in six-well plates, respectively. On the next day, the cells were treated with $40 \mathrm{nmol} \mathrm{T} 9(\mathrm{dR})$ and $10 \mathrm{nmol}$ cy 3 complex conjugated siGFP or $160 \mathrm{nmol}$ TP and $10 \mathrm{nmol}$ cy 3 complex conjugated siGFP (incubated at room temperature for 15 minutes). Cells in six-well plates were harvested at 24 hours and then washed with PBS. After being washed, the cells were permeabilized with FACS ${ }^{\text {TM }}$ permeabilizing solution 2, fixed in PBS with $1 \%$ formalin, and assayed by using Gallios flow cytometer (Beckman Coulter, Inc., Brea, CA, USA) with a minimum acquisition of 15,000 events and analyzed by using FlowJo software (TreeStar, Ashland, OR, USA).

\section{siRNA delivery and PR8 infection in vitro}

About $5 \times 10^{5}$ MDCK or A549 cells were seeded in sixwell plates and incubated at $37^{\circ} \mathrm{C}$ with $5 \% \mathrm{CO}_{2}$ overnight.
Subsequently, cells were treated with $80 \mathrm{nmol} \mathrm{T} 9(\mathrm{dR})$ and $20 \mathrm{nmol}$ siNP complex or $320 \mathrm{nmol} \mathrm{TP}$ and $20 \mathrm{nmol}$ siNP complex targeting influenza virus NP gene segment, and 20 nmol siGFP was used as control. At 6 hours posttreatment, the cells were infected with PR8 H1N1 influenza virus of multiplicity of infection $(\mathrm{MOI})=0.01$. At 24 hours post-infection, supernatant of cell culture was harvested for virus titration.

\section{siRNA delivery and PR8 challenge in vivo}

To assess the therapeutic effect of siNP delivered by T9(dR), 56 six-week-old female BALB/c mice were divided randomly into seven groups, eight mice in each group. Mice in group 1 were intravenously injected with $200 \mu \mathrm{L}$ of PBS as naïve control. Mice in groups 2 and 3 were given an intravenous injection of $50 \mathrm{nmol}$ siGFP with TP or T9(dR) in $200 \mu \mathrm{L}$ PBS supplemented with $5 \%$ glucose $(\mathrm{w} / \mathrm{v})$ to lessen pain in mice. Mice in groups 4, 5, 6, and 7 were intravenously injected with $10 \mathrm{nmol}$ or $50 \mathrm{nmol}$ siNP with T9(dR) in $200 \mu \mathrm{L}$ PBS supplemented with 5\% glucose. At 6 hours posttreatment with siNP, all mice were given an intranasal infection with $100 \mathrm{MLD}_{50}$ PR8 H1N1 influenza viruses in $50 \mu \mathrm{L}$ PBS. After being challenged with influenza virus, mice were observed daily and recorded for weight and clinical symptoms.

\section{Statistical analysis}

Comparisons between treated groups were performed by using a nonparametric one-way ANOVA with the Tukey multiple comparison test and Fisher's exact, and the final data were analyzed by using the log-rank test. The analyses were performed by using GraphPad Prism version 5.0 for Windows (GraphPad Software, Inc., La Jolla, CA, USA). $P$-values $<0.05$ were considered to be significant.

\section{Ethical statements}

In this study, animal experimental protocol was approved by the Institutional Animal Care and Use Committee at Qingdao Agricultural University. All animal experimental tests were carried out in accordance with the 2016 standards of laboratory animal in China and other related regulations in Animal Welfare Act.

\section{Results}

\section{Non-covalent nanoparticle formation}

In this study, T9(dR), GWTLNSAGYLLGKINLKA LAALAKKILdRdRdRdRdRdRdRdRdR, and TP were synthesized. T9(dR) or TP and siRNA formed non-covalent nanoparticles based on the phenomenon that positively charged peptides could condense negatively charged siRNA predominantly through electrostatic and hydrophobic 

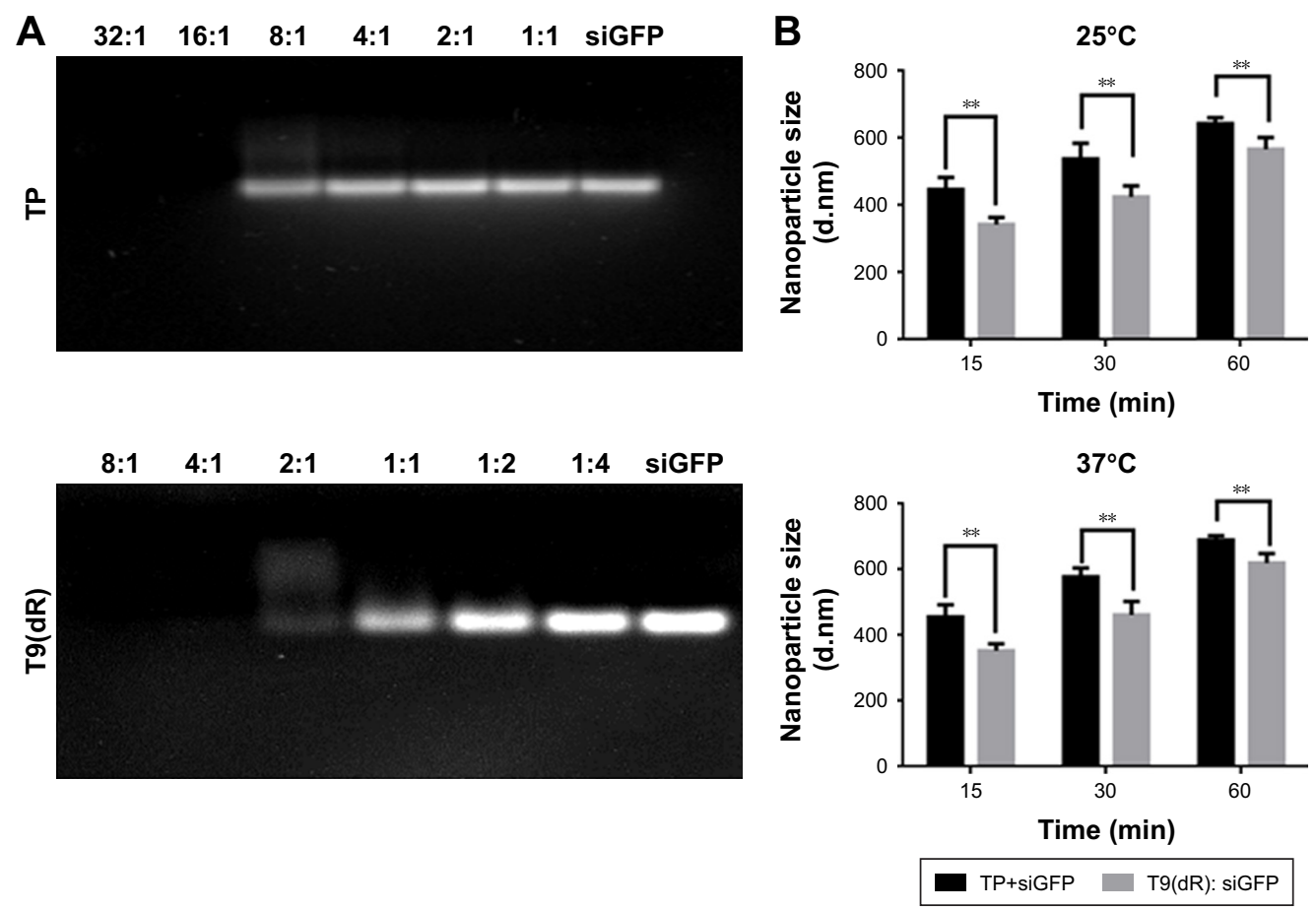

Figure I Cell-penetrating peptide T9(dR) or TP condensed siRNA and formed nanoparticles with siRNA. (A) Gel retardation assay and $(\mathbf{B})$ size of nanoparticles at $25^{\circ} \mathrm{C}$ and $37^{\circ} \mathrm{C}$. $* *$-values $<0.05$ were considered to be significant.

Abbreviation: TP, transportan.

interactions and form nanoparticles. ${ }^{34,35}$ To evaluate the avidity of T9(dR) or TP with siRNA, gel retardation assays were performed. Results in Figure 1A showed that when the mole ratio of $\mathrm{T} 9(\mathrm{dR})$ and siRNA against siGFP was $\geq 4: 1$, T9(dR) efficiently condensed siRNA and formed complexes, but the effective ratio of TP to siGFP is $\geq 16: 1$. Therefore, T9(dR) and siRNA were used in the ratio of $4: 1$ and TP and siRNA was used in ratio of 16:1 in follow-up experiments. To further assess the association rate of $\mathrm{T} 9(\mathrm{dR})$ or TP, siGFP and CPP were mixed in PBS and kept at $25^{\circ} \mathrm{C}$ or $37^{\circ} \mathrm{C}$ for 15,30 , and 60 minutes, and then the sample sizes were measured on Zetasizer Nano at $25^{\circ} \mathrm{C}$ or $37^{\circ} \mathrm{C}$. Results

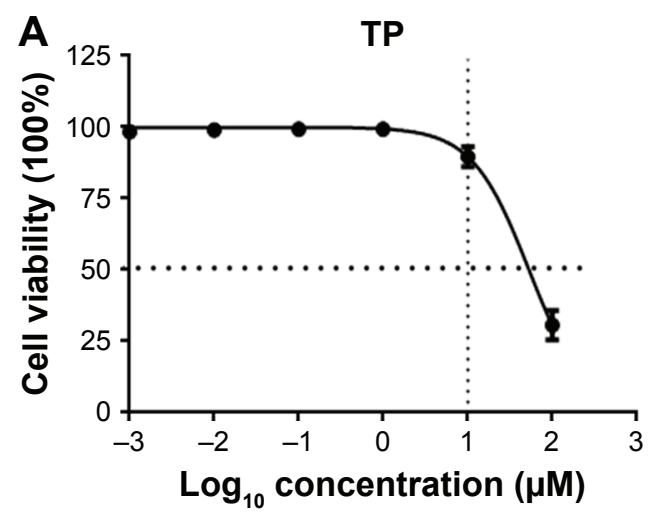

in Figure 1B showed that nanoparticles formed by $\mathrm{T} 9(\mathrm{dR})$ and siGFP are around $350 \mathrm{~nm}$ at 15 minutes and nanoparticles formed by TP and siGFP are around $450 \mathrm{~nm}(P<0.05)$. In addition, the size became larger as the time extended, and the dissociation of the nanoparticles at $37^{\circ} \mathrm{C}$ is faster than that at $25^{\circ} \mathrm{C}$.

\section{Lower toxicity of $\mathrm{T9}(\mathrm{dR})$ than TP}

Currently, most of the commercial siRNA transfection reagents are cationic lipids, which are with unbalanced toxicity to cells. ${ }^{36,37}$ We made a comparative analysis of the toxicity of T9(dR) and TP by using MTT assay. Results in Figure 2

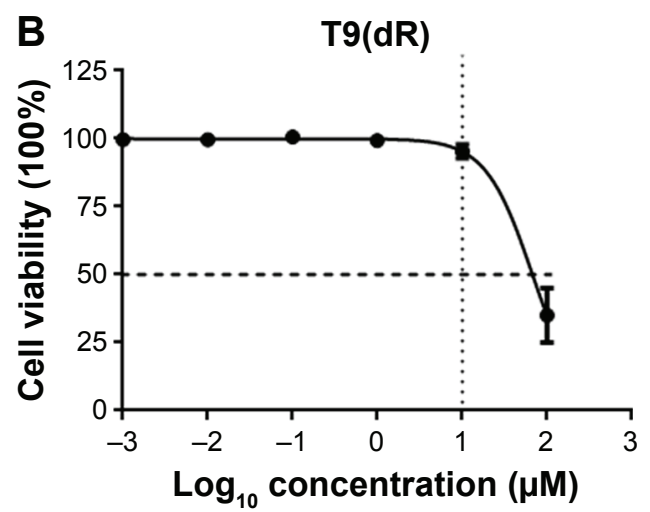

Figure 2 Cell viability treated with complex of T9(dR) or TP and tested by MTT assay. (A) T9(dR) and (B) TP. Abbreviation: TP, transportan. 
showed when $293 \mathrm{~T}$ cells in six-well plate were treated with $10 \mu \mathrm{mol}$ T9(dR) or TP, the cell viability was not substantially affected. Based on viability-regression curves, the CPP lethal doses sufficient to kill 50\% T9(dR)- or TP-treated cells were 85 and $90 \mu \mathrm{mol}$, respectively. These data could be a guide to further test $\mathrm{T} 9(\mathrm{dR})$ or TP.

\section{Higher delivery efficiency of $\mathrm{T} 9(\mathrm{dR})$ than TP}

To validate the efficient delivery by $\mathrm{T} 9(\mathrm{dR})$ of siRNA, transportation experiments were conducted on different cell lines with T9(dR) or TP and cy3-conjugated siRNA complex. Uptake of nanoparticles of $\mathrm{T} 9(\mathrm{dR})$ and siRNA by cells were indicated by cy3-positive cells. As expected, results in Figures 3 and 4 showed that T9(dR) efficiently transported siRNA into HEK 293T, A549, MDCK, and RAW cells. To further evaluate the delivery efficiency of
T9(dR), the delivery efficiencies of T9(dR) and TP were compared in different cell lines. Results in Figure 4 showed that T9(dR) had higher delivery efficiency than TP in all cell lines employed.

\section{siNP delivered by $T 9(\mathrm{dR})$ inhibited influenza virus replication in vitro}

To confirm siRNA delivered by $\mathrm{T} 9(\mathrm{dR})$ function properly, complex of siNP $(20 \mathrm{nmol})$ and T9(dR) or TP was incubated in MDCK cells or A459 cells. Subsequently, cells were infected with influenza virus of $\mathrm{MOI}=0.01$. At 24 hours post-infection, the supernatants were harvested. Virus titer in supernatant were titrated. In Figure 5, the results indicated that siNP delivered by $\mathrm{T} 9(\mathrm{dR})$ or TP inhibited the replication of PR8 influenza virus, but siNP delivered by T9(dR) has higher efficiency than TP. The results suggest that functional siRNA was transported into A549 and MDCK cells.
A
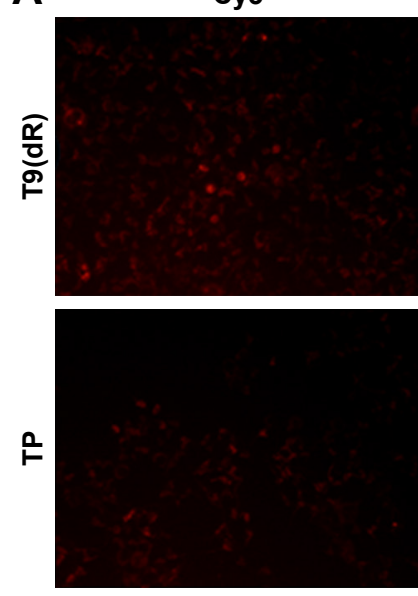

B
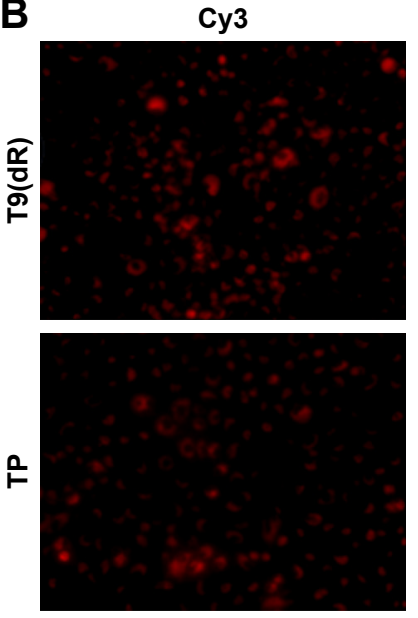

DAPI
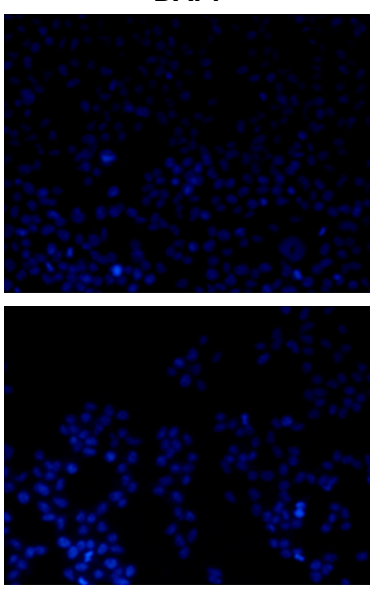

DAPI
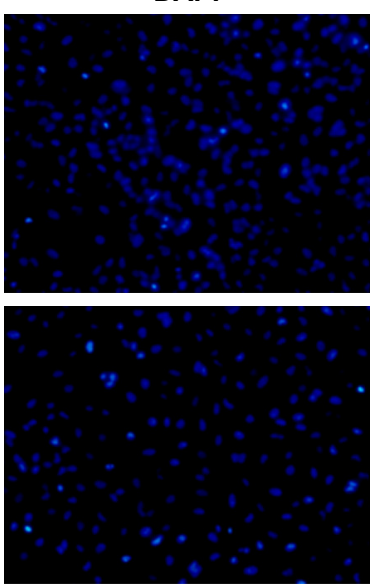

Merge
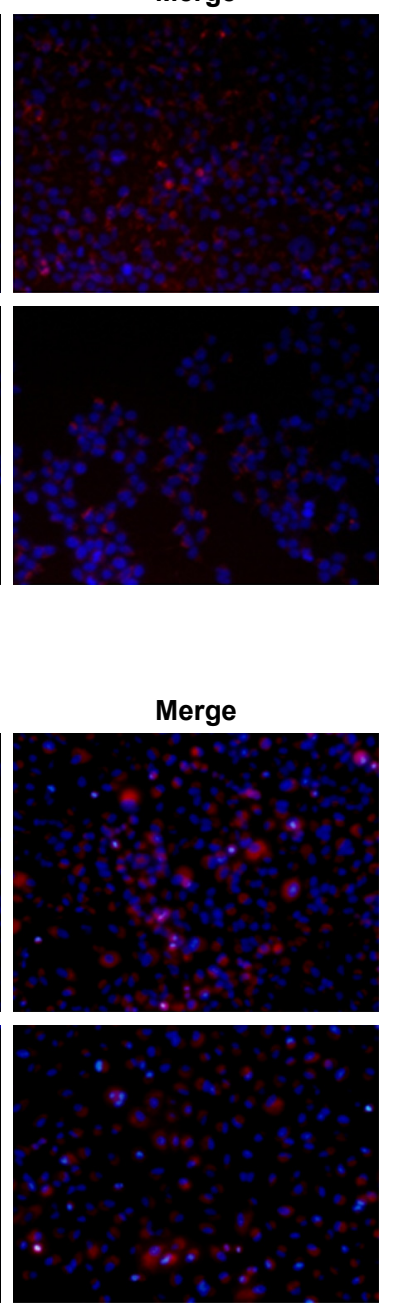

Figure 3 T9(dR) or TP transported siRNA into (A) 293T and (B) A549 cell lines. After T9(dR) or TP and cy3-conjugated siGFP were incubated for I5 minutes, the complex was transfected into cells seeded in eight-well chamber. At 24 hours post-transfection, cells were fixed, mounted with DAPI, and observed under a microscope (20X). Abbreviation: TP, transportan. 
A

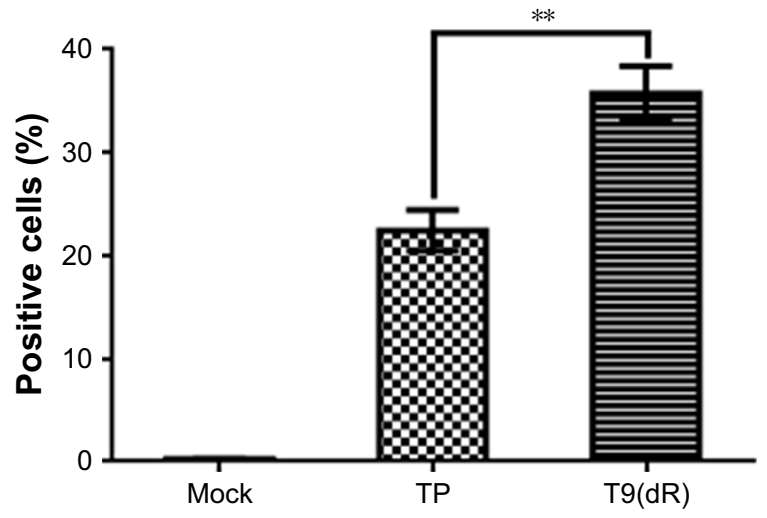

C

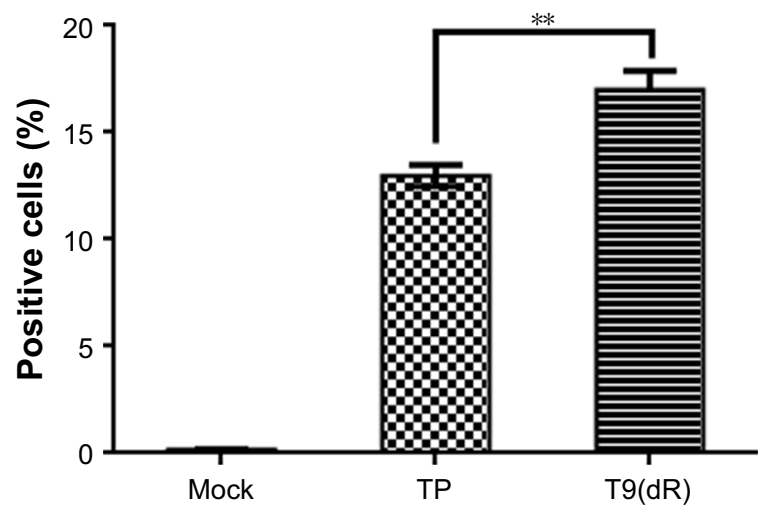

B

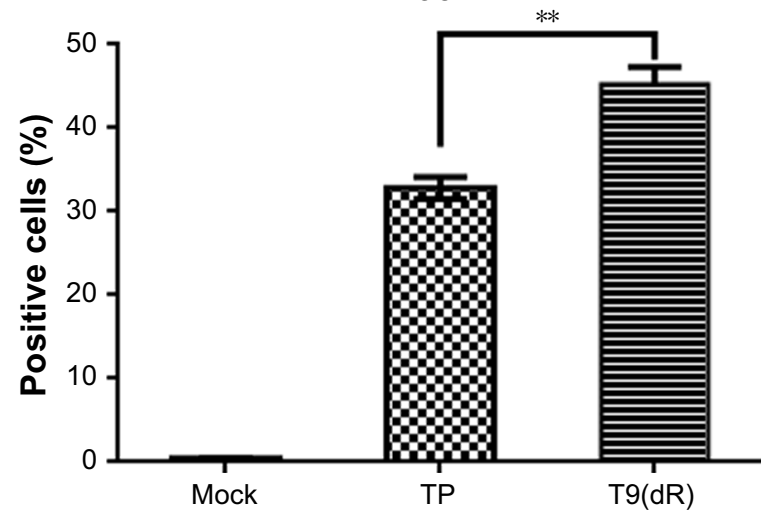

D

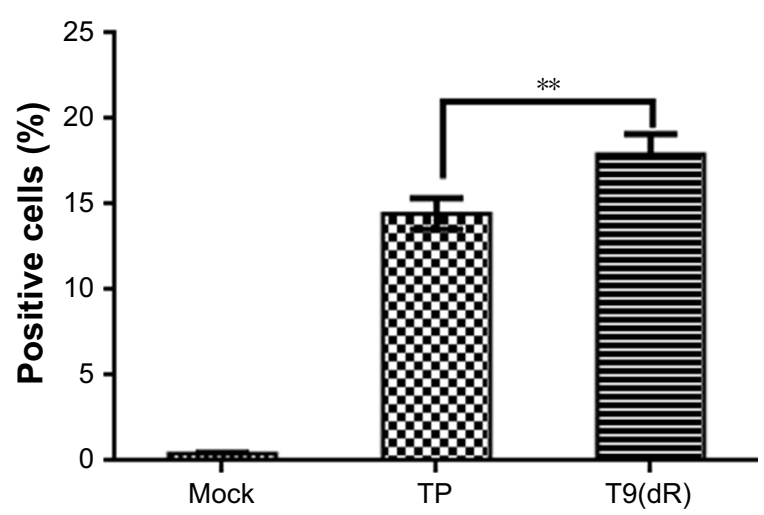

Figure 4 The delivery efficiency of T9(dR) with siRNA in A549, 293T, RAW, and MDCK cells (A-D). After T9(dR) and cy3-conjugated siGFP were incubated for I5 minutes, the complex was transfected into cells seeded in six-well plate. At 24 hours post-transfection, cells were run in a flow cytometer to detect cy3-positive cells. $* * P$-values $<0.05$ were considered to be significant.

Abbreviation: TP, transportan.

siRNA delivered by T9(dR) showed potent inhibition on influenza virus replication in mice

To test whether T9(dR) delivered siRNAs inhibit influenza virus replication in vivo, 6-week old $\mathrm{BALB} / \mathrm{c}$ mice were intravenously injected with complex of T9(dR) or TP with siGFP, or siNP. At 6 hours post-infection, the mice were infected with $100 \times \mathrm{MLD}_{50}$ PR8 H1N1 influenza virus. After infection, clinical symptoms and weight in mice were recorded every day. Mice treated with PBS or siGFP were
A

MDCK

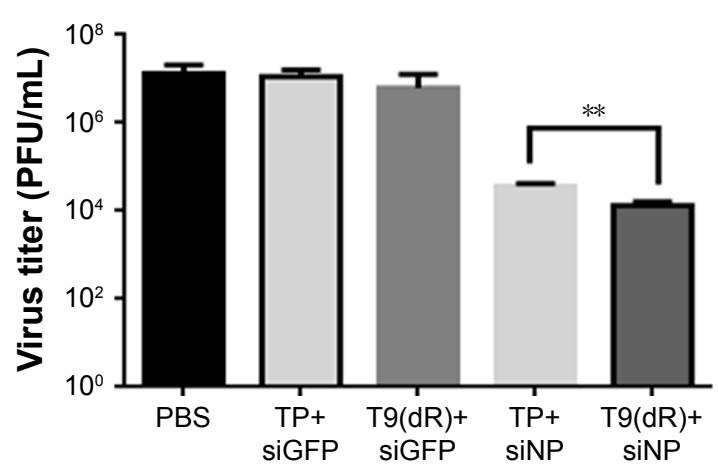

B

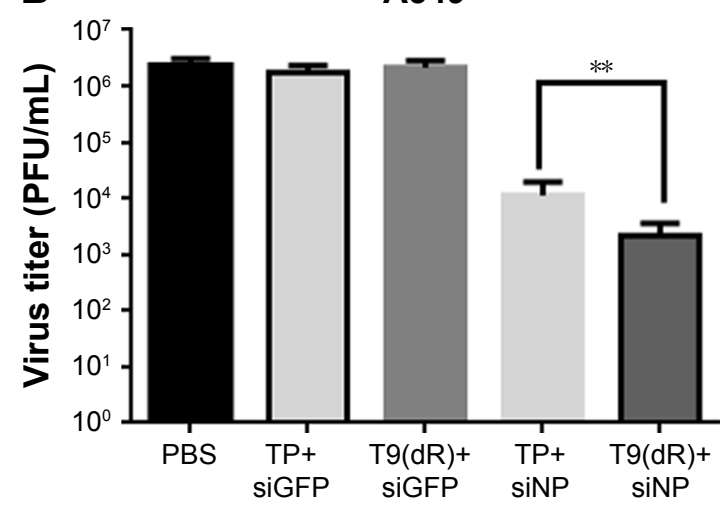

Figure 5 T9(dR) delivered functional siRNA into (A) MDCK and (B) A549 cell lines. MDCK and A549 cells were treated with siNP and infected with influenza virus of $\mathrm{MOI}=0.0 \mathrm{l}$. At 24 hours post-infection, viral titers in supernatant were titrated by standard plaque assay. ${ }^{* *} P$-values $<0.05$ were considered to be significant.

Abbreviations: MOI, multiplicity of infection; NP, nucleoprotein; TP, transportan. 
observed with poor growth, which included ruffled fur, and reduced activity with decreased food and water intake. Data in Figure 6 showed that mice administrated with PBS or siGFP experienced weight loss and death. Although the group injected with $50 \mathrm{nmol}$ siNP expressed just weight loss $(P$-value $<0.005)$, a complete recovery was observed on day 14 post-infection.

\section{Discussion}

Infection by human influenza A viruses incurs a huge burden to public health worldwide with an estimated 3-5 million hospitalization and 250,000-500,000 deaths every year. In the United States, around 200,000 infections with severe illness and 20,000 deaths annually were due to influenza A virus. Although influenza vaccines and anti-influenza drugs provide protection, their efficacies are inconsistent. Vaccination is an effective way to prevent infection by influenza viruses. Reportedly, there was 50\% mismatch between the seasonal influenza viruses and seasonal influenza vaccines from 1997 to $2005 .{ }^{38}$ In addition, the poor coverage of vaccination makes the prophylaxis of influenza ineffective. The mutation of neuraminidase (NA) also created a lot of oseltamivir-resistant influenza strains; ${ }^{39}$ hence, reinforcing development of new prophylactic and therapeutic tools is necessary according to the surveillance results of annual influenza epidemic.

A
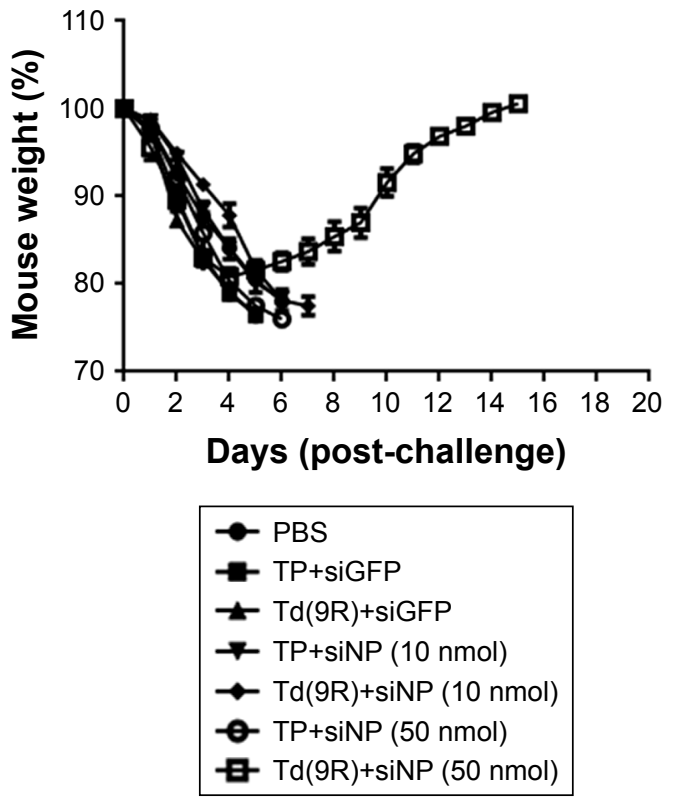

siRNA, as a potent tool to inhibit expression of target gene, has been investigated intensively in therapeutics. The siRNAs against conserved regions of the influenza gene segments are powerful promising tools to inhibit replication of influenza virus through degradation of target mRNA. ${ }^{14,40}$ However, naked siRNA was rapidly destroyed by serum nuclease. Due to the large size and anionic charge, the major impediment for therapeutic use of siRNAs is the lack of effective platform to deliver siRNA to desired cell types in vivo. ${ }^{41}$ Most of the current siRNA delivery implements are chemical regents and often exhibit unbalanced toxicity in both in vitro and in vivo trials. CPPs demonstrate a greater efficiency for the delivery of siRNAs. So far, many CPPs are identified from variable sources. Usually, the CPPs are 5-30 amino acids in length. However, how the length and structure affect the transportation ability of CPPs is still unknown. ${ }^{42}$ siRNAs binding to CPP form nanoparticles and confer resistance to nuclease degradation; however, the nanoparticles are driven by electrostatic and hydrophobic interaction creating instability in liquid environment. Thus, it is crucial to enhance the biological stability of the nanoparticles in biological fluids for a system with better delivery efficiency. Several methods were employed to increase the stability in vivo. ${ }^{43-46}$ In this study, we developed T9(dR) for effective delivery of siRNA in vivo. Initially, results of gel retardation showed that $\mathrm{T} 9(\mathrm{dR})$ and siRNA bind properly

B
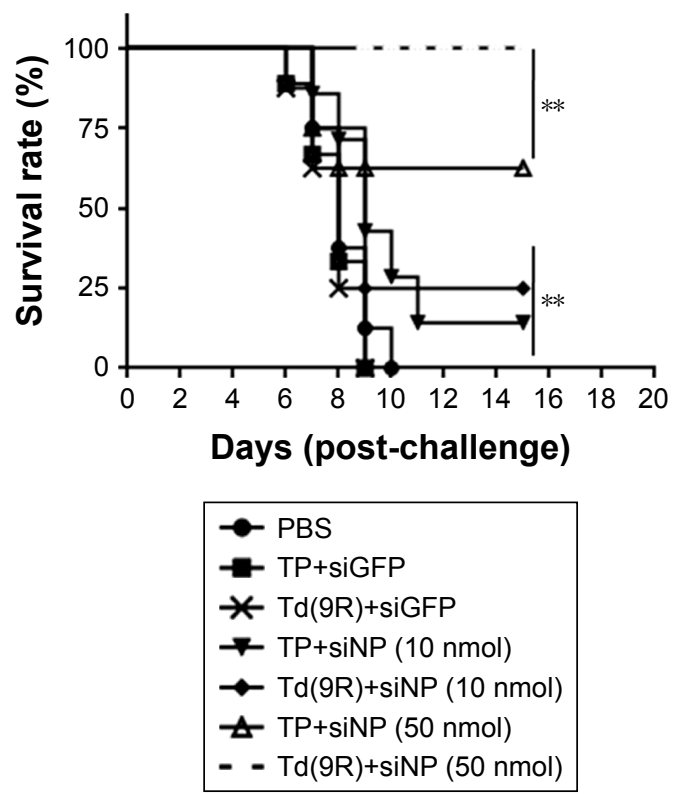

Figure 6 siNP transported by T9(dR) inhibited the replication of PR8 influenza virus in vivo. BALB/c mice were divided into seven groups, intravenously injected PBS, siGFP, siNP with T9(dR) or TP, and infected with influenza. Mouse weight was recorded $(\mathbf{A})$ and survival was calculated $(\mathbf{B})$. $* *$-values $<0.05$ were considered to be significant. Abbreviations: NP, nucleoprotein; TP, transportan. 
when the ratio was $>4: 1$ and the effective binding ratio of siGFP to TP is $16: 1$. Here, we did not know the binding mechanism of $\mathrm{T} 9(\mathrm{dR})$ and siRNA. Nevertheless, we hypothesized that it might be related to the charge and size of peptide. As a chimeric peptide, T9(dR) is longer than most of the published CPPs. The structure of CPPs is very complex. Correspondingly, T9(dR) and siNP formulated nanoparticles in large size around 350-600 nm within 1 hour at room temperature. Compared with the published results, the size is larger than the nanoparticles reported by the other researchers. At 15 and 60 minutes, average size of the particles increased from 350 to $650 \mathrm{~nm}$. It indicated that $\mathrm{T} 9(\mathrm{dR})$ and siRNA dissociated to some degree, but the mechanism is still under investigation. Nanoparticles comprising CPP and siRNA have to be attached to the cell membrane to intrude into cells. Usually, nanoparticle size is a key parameter for internalization pathways. In this study, TP or T9(dR) and siRNA complex may penetrate cellular membrane by caveolae-mediated internalization, which is the predominant entry route for $500-\mathrm{nm}$ particles. ${ }^{47}$ Otherwise, internalization of nanoparticles of size $<200 \mathrm{~nm}$ was observed to involve in clathrin-mediated endocytosis. ${ }^{48}$ Size of the nanoparticles also affect cytotoxicity upon internalization. Studies showed that smaller nanoparticles were highly toxic, caused rapid cell death, induced much higher levels of production of reactive oxygen species, and triggered NLRP3 inflammasome activation. ${ }^{49}$ However, large nanoparticles (>600 nm) induced strong membrane deformation during the internalization. ${ }^{50}$ Therefore, selection of nanoparticles with appropriate size is crucial for their medical application.

siRNA against highly conserved gene segment of influenza virus had been demonstrated to provide protection against a broad array of influenza strains, including H1N1, H5N1, H7N7, and H9N2 isolates. ${ }^{40,51}$ In this study, T9(dR) was used to deliver siRNA against NP gene segment. Results in vitro showed that $\mathrm{T} 9(\mathrm{dR})$ effectively transported siRNA into cytoplasm of MDCK, A549, RAW, and 293T cells. As for TP itself, it is an siRNA transporter with moderate efficiency, but attaching 9R to TP may increase siRNA uptake, allowed functional delivery of complexed siRNA, and conferred rapid siRNA translocation into the cytoplasm, but the mechanism is still under elucidation. ${ }^{23}$ It is possible that attachment of 9R to TP enhances the binding ability and stabilizes the cargo. CPPs showed lower toxicity as an siRNA delivery tool compared with viral vectors, but results published by Freire et al suggest that CPPs themselves perturb cellular pathways. ${ }^{52}$ Further investigation needs to be designed to test toxicity of $\mathrm{T} 9(\mathrm{dR})$ both in vitro and in vivo to assure a safe siRNA delivery platform in medical application.
We treated mice with complexes formed by $\mathrm{T} 9(\mathrm{dR})$ or TP and siNP before infection with PR8 H1N1 influenza virus. The results showed that siNP functioned well in vivo. It suggested that complexes formed by $\mathrm{T} 9(\mathrm{dR})$ and siNP were resistant to digestion in serum; however, we did not know whether treatment before infection influence the initiation of infection by influenza viruses. When $10 \mathrm{nmol}$ siNP gene was delivered into mouse lung by $\mathrm{T} 9(\mathrm{dR})$ or $\mathrm{TP}$, we observed partial protection. When $50 \mathrm{nmol}$ siNP was delivered into mouse lung, delivery efficiency of $\mathrm{T} 9(\mathrm{dR})$ was higher than that observed for TP. These results suggested that T9(dR) would be a promising functional siRNA delivery tool. In this study, only siRNA against the NP gene segment was employed. In future investigation, mixture of siRNA against the NP, M, and polymerase gene segments will be tested to improve the inhibition on influenza viruses. Perhaps, the cocktail siRNAs will enhance the protection against influenza viruses.

\section{Conclusion}

In this study, we designed and synthesized a TP-derived CPP, T9(dR). Results of in vitro and in vivo trials showed T9(dR) delivered siRNA with higher efficiency as compared to delivery by TP. This study indicated that $\mathrm{T} 9(\mathrm{dR})$ would prove to be a promising nucleotide delivery tool with potential application for nucleotide drug and vaccine.

\section{Acknowledgments}

This study was supported by the Priority Academic Talent Team Cultivation Program of Shandong Colleges and Universities and Shandong National Science Foundation (ZR2017MC002).

\section{Disclosure}

All authors report no conflicts of interest in this work.

\section{References}

1. Thompson WW, Shay DK, Weintraub E, et al. Mortality associated with influenza and respiratory syncytial virus in the United States. JAMA. 2003;289(2):179-186.

2. Dushoff J, Plotkin JB, Viboud C, Earn DJ, Simonsen L. Mortality due to influenza in the United States-an annualized regression approach using multiple-cause mortality data. Am J Epidemiol. 2006;163(2):181-187. doi:10.1093/aje/kwj024

3. Fraser C, Donnelly CA, Cauchemez S, et al. Pandemic potential of a strain of influenza A (H1N1): early findings. Science. 2009;324(5934): 1557-1561. doi:10.1126/science.1176062

4. Dobrovolny HM, Beauchemin CAA. Modelling the emergence of influenza drug resistance: the roles of surface proteins, the immune response and antiviral mechanisms. PLoS One. 2017;12(7):e0180582. doi:10.1371/ journal.pone. 0180582

5. Nicholson KG, Aoki FY, Osterhaus AD, et al. Efficacy and safety of oseltamivir in treatment of acute influenza: a randomised controlled trial. Neuraminidase inhibitor flu treatment investigator group. Lancet. 2000;355(9218):1845-1850. 
6. Aoki FY, Macleod MD, Paggiaro P, et al. Early administration of oral oseltamivir increases the benefits of influenza treatment. J Antimicrob Chemother. 2003;51(1):123-129.

7. Li Z, Watanabe T, Hatta M, et al. Mutational analysis of conserved amino acids in the influenza A virus nucleoprotein. J Virol. 2009;83(9): 4153-4162. doi:10.1128/JVI.02642-08

8. Elbashir SM, Harborth J, Lendeckel W, Yalcin A, Weber K, Tuschl T. Duplexes of 21-nucleotide RNAs mediate RNA interference in cultured mammalian cells. Nature. 2001;411(6836):494 498. doi:10.1038/35078107

9. Hammond SM, Bernstein E, Beach D, Hannon GJ. An RNA-directed nuclease mediates post-transcriptional gene silencing in drosophila cells. Nature. 2000;404(6775):293-296. doi:10.1038/35005107

10. Nishikura K. A short primer on RNAi: RNA-directed RNA polymerase acts as a key catalyst. Cell. 2001;107(4):415-418.

11. Song Y, Feng Y, Leblanc MH, Castagloni N Jr, Liu YM. 1-Benzyl1,2,3,4-tetrahydroisoquinoline passes through the blood-brain barrier of rat brain: an in vivo microdialysis study. Neurosci Lett. 2006;395(1): 63-66. doi:10.1016/j.neulet.2005.10.050

12. Ge Q, Filip L, Bai A, Nguyen T, Eisen HN, Chen J. Inhibition of influenza virus production in virus-infected mice by RNA interference. Proc Natl Acad Sci US A. 2004;101(23):8676-8681. doi:10.1073/pnas.0402486101

13. Rajput R, Khanna M, Kumar P, et al. Small interfering RNA targeting the nonstructural gene 1 transcript inhibits influenza A virus replication in experimental mice. Nucleic Acid Ther. 2012;22(6):414-422. doi:10.1089/nat.2012.0359

14. Lin L, Liu Q, Berube N, Detmer S, Zhou Y. 5'-Triphosphate-short interfering RNA: potent inhibition of influenza A virus infection by gene silencing and RIG-I activation. J Virol. 2012;86(19):10359-10369. doi:10.1128/JVI.00665-12

15. Derossi D, Chassaing G, Prochiantz A. Trojan peptides: the penetratin system for intracellular delivery. Trends Cell Biol. 1998;8(2):84-87.

16. Sanchez-Navarro M, Teixido M, Giralt E. Jumping hurdles: peptides able to overcome biological barriers. Acc Chem Res. 2017;50: 1847-1854. doi:10.1021/acs.accounts.7b00204

17. Subramanya S, Kim SS, Abraham S, et al. Targeted delivery of small interfering RNA to human dendritic cells to suppress dengue virus infection and associated proinflammatory cytokine production. $J$ Virol. 2010;84(5):2490-2501. doi:10.1128/JVI.02105-08

18. Subramanya S, Armant M, Salkowitz JR, et al. Enhanced induction of HIV-specific cytotoxic T lymphocytes by dendritic cell-targeted delivery of SOCS-1 siRNA. Mol Ther. 2010;18(11):2028-2037. doi:10.1038/ mt.2010.148

19. Barany-Wallje E, Gaur J, Lundberg P, Langel U, Graslund A. Differential membrane perturbation caused by the cell penetrating peptide Tp 10 depending on attached cargo. FEBS Lett. 2007;581(13):2389-2393. doi:10.1016/j.febslet.2007.04.046

20. Shukla RS, Jain A, Zhao Z, Cheng K. Intracellular trafficking and exocytosis of a multi-component siRNA nanocomplex. Nanomedicine. 2016;12(5):1323-1334. doi:10.1016/j.nano.2016.02.003

21. ZhuP,JinL. Cell penetrating peptides: a promising tool for the cellularuptake of macromolecular drugs. Curr Protein Pept Sci. 2018;19(2):211-220. doi:10.2174/1389203718666170710115240

22. Parnaste L, Arukuusk P, Langel K, Tenson T, Langel U. The formation of nanoparticles between small interfering RNA and amphipathic cell-penetrating peptides. Mol Ther Nucleic Acids. 2017;7:1-10 doi:10.1016/j.omtn.2017.02.003

23. Zeller S, Choi CS, Uchil PD, et al. Attachment of cell-binding ligands to arginine-rich cell-penetrating peptides enables cytosolic translocation of complexed siRNA. Chem Biol. 2015;22(1):50-62. doi:10.1016/j. chembiol.2014.11.009

24. Pooga M, Hallbrink M, Zorko M, Langel U. Cell penetration by transportan. FASEB J. 1998;12(1):67-77.

25. Wierzbicki PM, Kogut-Wierzbicka M, Ruczynski J, et al. Protein and siRNA delivery by transportan and transportan 10 into colorectal cancer cell lines. Folia Histochem Cytobiol. 2014;52(4):270-280. doi:10.5603/ FHC.a2014.0035

26. Soomets U, Lindgren M, Gallet X, et al. Deletion analogues of transportan. Biochim Biophys Acta. 2000;1467(1):165-176.
27. Anko M, Majhenc J, Kogej K, et al. Influence of stearyl and trifluoromethylquinoline modifications of the cell penetrating peptide TP10 on its interaction with a lipid membrane. Biochim Biophys Acta. 2012; 1818(3):915-924. doi:10.1016/j.bbamem.2011.12.028

28. Liu BR, Lin MD, Chiang HJ, Lee HJ. Arginine-rich cell-penetrating peptides deliver gene into living human cells. Gene. 2012;505(1):37-45. doi:10.1016/j.gene.2012.05.053

29. Kim SS, Ye C, Kumar P, et al. Targeted delivery of siRNA to macrophages for anti-inflammatory treatment. Mol Ther. 2010;18(5): 993-1001. doi:10.1038/mt.2010.27

30. Nakase I, Noguchi K, Aoki A, Takatani-Nakase T, Fujii I, Futaki S. Arginine-rich cell-penetrating peptide-modified extracellular vesicles for active macropinocytosis induction and efficient intracellular delivery. Sci Rep. 2017;7(1):1991. doi:10.1038/s41598-017-02014-6

31. Ye C, Choi JG, Abraham S, et al. Human macrophage and dendritic cell-specific silencing of high-mobility group protein $\mathrm{B} 1$ ameliorates sepsis in a humanized mouse model. Proc Natl Acad Sci U S A. 2012;109(51):21052-21057. doi:10.1073/pnas.1216195109

32. Jain A, Barve A, Zhao Z, Jin W, Cheng K. Comparison of avidin, neutravidin, and streptavidin as nanocarriers for efficient siRNA delivery. Mol Pharm. 2017;14(5):1517-1527. doi:10.1021/acs. molpharmaceut.6b00933

33. Zhao Z, Li Y, Jain A, et al. Development of a peptide-modified siRNA nanocomplex for hepatic stellate cells. Nanomedicine. 2018;14(1): 51-61. doi:10.1016/j.nano.2017.08.017

34. Deshayes S, Morris M, Heitz F, Divita G. Delivery of proteins and nucleic acids using a non-covalent peptide-based strategy. Adv Drug Deliv Rev. 2008;60(4-5):537-547. doi:10.1016/j.addr.2007.09.005

35. Meade BR, Dowdy SF. Enhancing the cellular uptake of siRNA duplexes following noncovalent packaging with protein transduction domain peptides. Adv Drug Deliv Rev. 2008;60(4-5):530-536. doi:10.1016/ j.addr.2007.10.004

36. Lv H, Zhang S, Wang B, Cui S, Yan J. Toxicity of cationic lipids and cationic polymers in gene delivery. J Control Release. 2006;114(1): 100-109. doi:10.1016/j.jconrel.2006.04.014

37. Morille M, Passirani C, Vonarbourg A, Clavreul A, Benoit JP. Progress in developing cationic vectors for non-viral systemic gene therapy against cancer. Biomaterials. 2008;29(24-25):3477-3496. doi:10.1016/ j.biomaterials.2008.04.036

38. Weaver EA, Rubrum AM, Webby RJ, Barry MA. Protection against divergent influenza H1N1 virus by a centralized influenza hemagglutinin. PLoS One. 2011;6(3):e18314. doi:10.1371/journal.pone. 0018314

39. Quiliano M, Valdivia-Olarte H, Olivares C, et al. Molecular distribution of amino acid substitutions on neuraminidase from the 2009 (H1N1) human influenza pandemic virus. Bioinformation. 2013;9(13):673-679. doi: $10.6026 / 97320630009673$

40. Ge Q, McManus MT, Nguyen T, et al. RNA interference of influenza virus production by directly targeting mRNA for degradation and indirectly inhibiting all viral RNA transcription. Proc Natl Acad Sci US A. 2003;100(5):2718-2723. doi:10.1073/pnas.0437841100

41. Kumar P, Wu H, McBride JL, et al. Transvascular delivery of small interfering RNA to the central nervous system. Nature. 2007;448(7149): 39-43. doi:10.1038/nature05901

42. Collado Camps E, Brock R. An opportunistic route to success: towards a change of paradigm to fully exploit the potential of cell-penetrating peptides. Bioorg Med Chem. 2018;26(10):2780-2787. doi:10.1016/j. bmc.2017.11.004

43. Crombez L, Morris MC, Dufort S, et al. Targeting cyclin B1 through peptide-based delivery of siRNA prevents tumour growth. Nucleic Acids Res. 2009;37(14):4559-4569. doi:10.1093/nar/gkp451

44. Kim YW, Grossmann TN, Verdine GL. Synthesis of all-hydrocarbon stapled alpha-helical peptides by ring-closing olefin metathesis. Nat Protoc. 2011;6(6):761-771. doi:10.1038/nprot.2011.324

45. Lehto T, Abes R, Oskolkov N, et al. Delivery of nucleic acids with a stearylated (RxR)4 peptide using a non-covalent co-incubation strategy. J Control Release. 2010;141(1):42-51. doi:10.1016/j.jconrel.2009. 08.028 
46. Majumder P, Bhunia S, Chaudhuri A. A lipid-based cell penetrating nano-assembly for RNAi-mediated anti-angiogenic cancer therapy. Chem Commun (Camb). 2018;54(12):1489-1492. doi:10.1039/ c7cc08517f

47. Shang L, Nienhaus K, Nienhaus GU. Engineered nanoparticles interacting with cells: size matters. J Nanobiotechnology. 2014;12:5. doi:10.1186/ 1477-3155-12-5

48. Mironava T, Hadjiargyrou M, Simon M, Jurukovski V, Rafailovich MH. Gold nanoparticles cellular toxicity and recovery: effect of size, concentration and exposure time. Nanotoxicology. 2010;4(1):120-137. doi: $10.3109 / 17435390903471463$

49. Lunov O, Syrovets T, Loos C, et al. Amino-functionalized polystyrene nanoparticles activate the NLRP3 inflammasome in human macrophages. ACS Nano. 2011;5(12):9648-9657. doi:10.1021/nn203596e
50. Zhao Y, Sun X, Zhang G, Trewyn BG, Slowing II, Lin VS. Interaction of mesoporous silica nanoparticles with human red blood cell membranes: size and surface effects. ACS Nano. 2011;5(2):1366-1375. doi:10.1021/ $\mathrm{nn} 103077 \mathrm{k}$

51. Tompkins SM, Lo CY, Tumpey TM, Epstein SL. Protection against lethal influenza virus challenge by RNA interference in vivo. Proc Natl Acad Sci U S A. 2004;101(23):8682-8686. doi:10.1073/pnas. 0402630101

52. Freire JM, Rego de Figueiredo I, Valle J, et al. siRNA-cell-penetrating peptides complexes as a combinatorial therapy against chronic myeloid leukemia using BV173 cell line as model. J Control Release. 2017;245: 127-136. doi:10.1016/j.jconrel.2016.11.027

\section{Publish your work in this journal}

Drug Design, Development and Therapy is an international, peerreviewed open-access journal that spans the spectrum of drug design and development through to clinical applications. Clinical outcomes, patient safety, and programs for the development and effective, safe, and sustained use of medicines are the features of the journal, which has also been accepted for indexing on PubMed Central. The manuscript management system is completely online and includes a very quick and fair peer-review system, which is all easy to use. Visit http://www.dovepress.com/testimonials.php to read real quotes from published authors.

Submit your manuscript here: http://www.dovepress.com/drug-design-development-and-therapy-journal 